\title{
Grading quality of total mesorectal excision specimen by surgeons
}

\section{Maharjan DK', Thapa PB²}

'Dhiresh Kumar Maharjan, Lecturer; ${ }^{2}$ Prabin Bikram Thapa, Professor; Department of Surgery, Kathmandu Medical College Teaching Hospital, Sinamangal, Kathmandu, Nepal.

\begin{abstract}
Background: Total mesorectal excision has been gold standard since 1978. But standardization of surgery with quality assurance of total mesorectal excision specimen has been a challenging issue in developing countries. However, quality of macroscopic total mesorectal excision can be graded immediately by operating surgeon before specimen has been fixed in formalin and photographic documentation of gross specimen by surgeons is possible and practical.

Objective: To grade macroscopic total mesorectal excision specimen by surgeon and document it photographically and compare it with reporting received from pathologist.

Methods: A prospective observational study conducted from Jan 2014 to Jan 2016 at Department of Surgery, Kathmandu Medical College Teaching Hospital, Kathmandu, Nepal. All consecutive patients with rectal cancer (upper/middle and lower) without distant metastasis were included.Immediate after surgery,macroscopic specimen of TME were graded by operating surgeon and photo-documentation with one anterior, one posterior and two right and left lateral views of total mesorectal excision photos were taken and documented with printed form along with operative notes.

Results: There were 40 patients with rectal cancer who underwent surgery during this period. Among those patients, the median age was 25 years of which $30 \%$ were females. Twenty-four patients underwent low anterior resection whereas thirteen had ultralow anterior resection and three had abdominal perineal resection. All patients had photo documentation.Complete mesorectal excision was seen in 36 patients and four patients had near complete total mesorectal excision when graded by surgeons. However, pathologist reported six (16.6\%) patients having near complete mesorectum among those which had been graded as complete by surgeons.

Conclusion: Grading of macroscopic total mesorectal excision specimen by surgeon is feasible and with use of photographic documentation, it can help to assess the quality of surgeons work and can be a good tool for feedback for surgeons to improve.
\end{abstract}

Key words: Photo-documentation, Rectal cancer, Total Mesorectal Excision grade

\section{INTRODUCTION}

ince the introduction of total mesorectal excision $\circlearrowleft$ (TME) for rectal cancer by Sir Bill Heald in 1978, there has been a significant decrease in local recurrence from $38 \%$ to $8 \% \%^{1,2}$ and with the use of neoadjuvant radiotherapy and chemotherapy (neo $\mathrm{RT} / \mathrm{CT}$ ) this rate has further decreased to $2 \%{ }^{3,4}$.

With this significant advantage of neoadjuvant RT/ $\mathrm{CT}$ and TME procedure, there have been many issues regarding how it can be standardized in a developing

\section{Address for correspondence}

Dr. Dhiresh Kumar Maharjan MRCSEd, FCPS

Lecturer, Department of Surgery

Kathmandu Medical College Teaching Hospital

Sinamangal, Kathmandu, Nepal

E-mail: maharjandhiresh@gmail.com country like Nepal. Prof. Phil Quirke has designed the concept of photographic documentation of pathological specimen before formalin preservation ${ }^{5}$ which has been incorporated in other countries like Belgium as Procare Protocol with significant change in quality control of surgery and its outcome ${ }^{6}$.

Hence, our objective is to grade macroscopic TME specimen by operating surgeon and document it photographically immediately after surgery and to compare with final pathological report given by pathologists.

\section{METHODS}

This was a prospective observational study carried out for duration of 2 years (Jan 2014 to Jan 2016) at the Department of Surgery, Kathmandu Medical College 
Teaching Hospital (KMCTH), Nepal. Ethical approval was taken from Institutional Review Committee of KMCTH before commencing study and a proper informed consent was taken from the patients.

All patients who had undergone low anterior resection (LAR) for upper and middle rectal cancer, ultralow anterior resection (Ultra LAR) for lower rectal cancer, and abdomino-perineal resection(APR) for very low rectal cancer were included in the study.

Patients with recto sigmoid junction cancer who had undergone anterior resection with partial mesorectal excision(PME)were excluded.

As per KMCTH departmental surgical malignancy audit 2012, there were 15 cases of carcinoma rectum that had undergone surgery at the Department of Surgery. Hence, we expect a minimum of 10 cases of carcinoma of rectum per year. Statistical Package for Social Sciences (SPSS) version 16 was used. Descriptive variables were mentioned as frequency and represented in percentage. All patients with carcinoma of rectum (upper,middle and lower) were included consecutively. All patients had neoadjuvant long course concurrent chemoradiotherapy of $50 \mathrm{~Gy}$ in 28 fractions to the pelvis for five and half weeks and concurrent capcitabine $825 \mathrm{mg} / \mathrm{m} 2$ twice daily 5 days a week for a period of five and half weeks and surgery was done after 4-6 weeks ${ }^{7,8}$.

Grading of macroscopic total mesorectal excision specimen were done by operating surgeon immediately after surgery according toTable 1 which outlines the approach by Prof. Philip Quirke from Leeds University for the assessment of the TME specimen 9,10 and postoperative photographic documentation was done. Then all specimens were sent to pathology department for macroscopic and microscopic evaluation of TME without formalin fixation and later compared with final pathological biopsy report. The printed photographic documentation of TME specimen (Figure1, 2 \& 3) were kept along with patient discharge file for later audit purpose.

\section{RESULTS}

There were a total of 40 patients who underwent surgery during this time period.

Table 1: Grading of Macroscopic TME specimen

\section{Grade of Mesorectum}

Mesorectal plane (complete)

Intramesorectal plane

(nearly complete)

Muscularis propria plane (incomplete)

Sphincteric complex

Extralevator

Sphincteric plane

Intrasphincteric/submucosal plane
Intact mesorectum with only minor irregularities

No defects deeper than $5 \mathrm{~mm}$

No coning toward the distal margin of the resection specimen

Moderate bulk to the mesorectum

One or more defects greater than $5 \mathrm{~mm}$ deep within the mesorectum

Moderate coning

No visible muscularis propria

Exposed muscularis propria

Moderate to marked coning

Cylindrical specimen with no waist effect

Levators removed en bloc

Slight waist effect

No significant defects or perforations

Significant waist effect

Perforation or missing areas of muscularis propria 
Table 2: Demographic characteristics of patient

\begin{tabular}{|c|c|c|}
\hline Variables & Number(n) & Percentage (\%) \\
\hline \multicolumn{3}{|l|}{ Age group (Median : 25years) } \\
\hline Less than 25 years & 22 & 55 \\
\hline $25-50$ years & 10 & 25 \\
\hline More than 50 years & 8 & 20 \\
\hline \multicolumn{3}{|l|}{ Gender } \\
\hline Male & 28 & 70 \\
\hline Female & 12 & 30 \\
\hline \multicolumn{3}{|l|}{ Pathologic type } \\
\hline Well differentiated Adenocarcinoma & 20 & 50 \\
\hline Moderately differentiated Adenocarcinoma & 2 & 5 \\
\hline Poorly differentiated Adenocarcinoma & 18 & 45 \\
\hline \multicolumn{3}{|l|}{ Distance from anal verge } \\
\hline More than $5 \mathrm{~cm}$ & 24 & 60 \\
\hline Less than $5 \mathrm{~cm}$ & 16 & 40 \\
\hline \multicolumn{3}{|l|}{ Tumor location } \\
\hline Anterior & 8 & 20 \\
\hline Posterior & 6 & 15 \\
\hline Circumferential & 24 & 60 \\
\hline Lateral & 2 & 5 \\
\hline \multicolumn{3}{|l|}{ Pathological response post Neoadjuvant } \\
\hline Complete pathological response & 2 & 5 \\
\hline Partial pathological response & 33 & 82.5 \\
\hline No pathological response & 5 & 12.5 \\
\hline \multicolumn{3}{|l|}{ Surgery } \\
\hline Low anterior resection & 24 & 60 \\
\hline Ultralow anterior resection & 13 & 32.5 \\
\hline Abdomino-perineal resection & 3 & 7.5 \\
\hline \multicolumn{3}{|l|}{ Mode of surgery } \\
\hline Laparoscopic & 6 & 15 \\
\hline Laparoscopic assisted & 26 & 65 \\
\hline Open & 8 & 20 \\
\hline
\end{tabular}

Table 3: Surgeon grading versus pathologist grading of TME

\begin{tabular}{lcc}
\hline & Surgeon grading of TME & Pathologist grading of TME \\
\hline Complete TME & 36 & 30 \\
Nearly complete TME & 4 & 10 \\
\hline
\end{tabular}




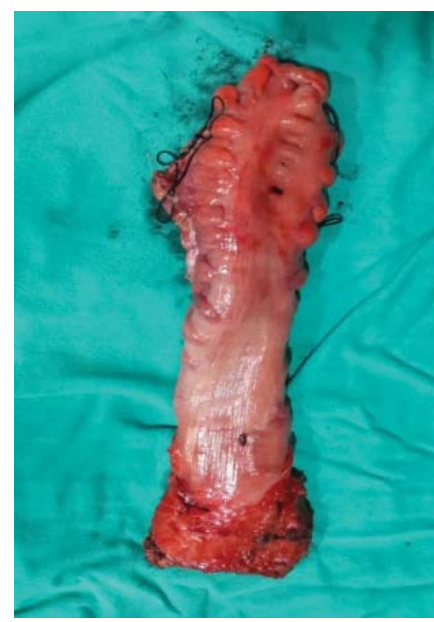

Figure 1: Anterior view

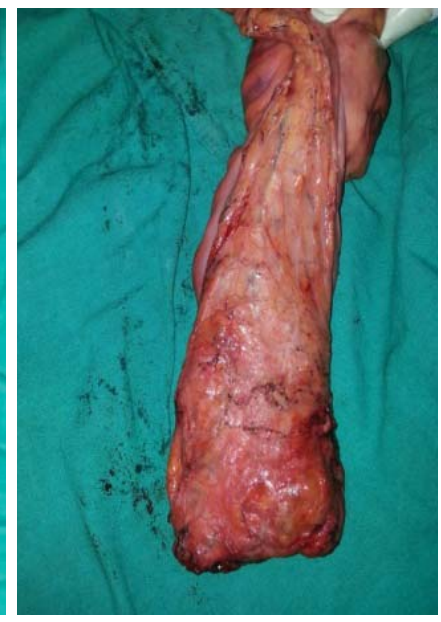

Figure 2: Posterior view

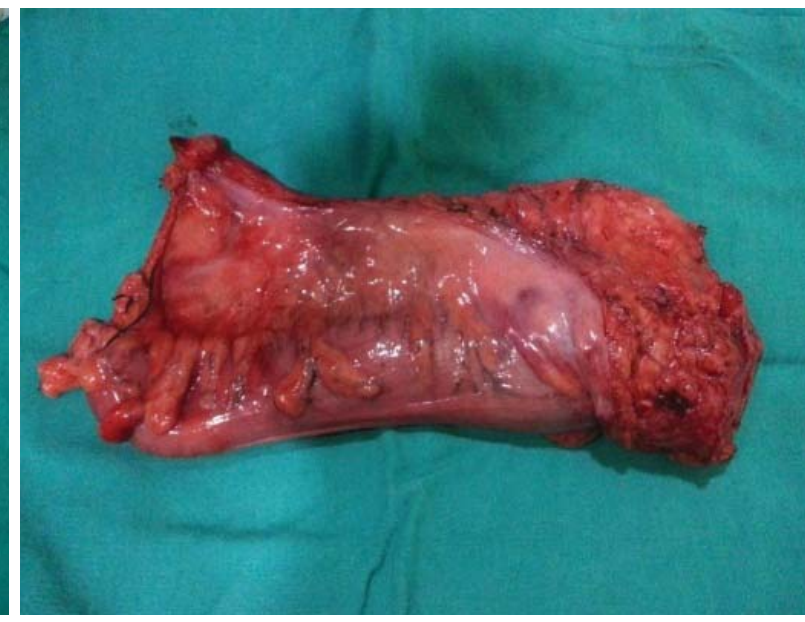

Figure 3: Lateral view

\section{DISCUSSION}

As prevention of local recurrence is best achieved by proper TME, Prof.Philip Quirke stated that origin of most pelvic recurrence after conventional surgery was because of inadequate resection of the mesorectum mainly violated circumferential margins ${ }^{11}$. Hence, how can qualities of a good TME be assured?

Knowledge of grading of total mesorectal excision is equally important for surgeon beside pathologist as it helps to refine the approach of total mesorectal excision during surgery and pursues' surgeon to achieve the highest standard of quality surgery. This assurance of quality surgery can be documented with the help of photographs of specimen before formalin fixation which in later date can be used as tool for clinicopathological audit. In our study, 36 patients had complete TME according to surgeon's grading. Among them, six patients $(16.6 \%)$ seemed to have near complete TME when graded by pathologist. A study by Peter Bond even ${ }^{12}$ has shown that among 136 specimens, 54\% of the specimens had discernable volume defects in the mesorectum when revaluated by the pathologist on standard photographic documentation. When these observations were correlated with the prospective macroscopic assessment of the specimen with regards to the plane of surgery achieved, $42 \%$ observable volume defects were seenin the mesorectum despite being initially graded to be in complete mesorectal plane.
Another advantage is that it helps to standardize study protocol among confronting factors to decrease the biasness as photographs can be an objective model for quality control ${ }^{13}$.

Besides that, local recurrence depends upon quality of TME which has shown that incomplete TME have local recurrence and was $41 \%$,whereas in near complete it was $6 \%$ and in complete TME the rate was less than $2 \%{ }^{14}$.

Similarly, Nagtegaal et al have shown that local and distant recurrence rate was significantly higher among incomplete TME group $36.1 \%$ vs $20.3 \%$ recurrence in the group with a complete mesorectum $(P=0.02)^{15}$.

Photographic documentation may be helpful in predicting local recurrence, deciding in post adjuvant therapy, observing the practicality of re-resection if there is local recurrence andhence can help in triaging the treatment.

\section{CONCLUSION}

Grading of total mesorectal excision by surgeon is necessary to assure their quality of surgery which can be documented by photography. This document can help to assess and audit the surgeons work quality when we follow up in long term.

\section{REFERENCES}

1. Heald RJ, Moran BJ, Ryall RDH, Sexton R, MacFarlane JK. The Basingstoke Experience of Total Mesorectal Excision, 1978-1997. Arch Surg.1998; 133(8):894-8. DOI: 10.1001/archsurg.133.8.894

2. Havenga K, Enker WE, Norstein J, Moriya Y, Heald RJ, Van Houwelingen $\mathrm{HC}$, et al. Improved survival and local control after total mesorectal excision or D3 lymphadenectomy in the treatment of primary rectal 
cancer: an international analysis of 1411 patients. Eur J Surg Onco. 1999; 25(4):368-74. DOI:10.1053/ ejso.1999.0659.

3. Van GW, Marijnen CA, Nagtegaal ID, Kranenbarg EM, Putter $\mathrm{H}$, Wiggers $\mathrm{T}$, et al. Preoperative radiotherapy combined with total mesorectal excision for resectable rectal cancer: 12-year followup of the multicentre randomised controlled TME trial. Lancet Onco. 2011; 12(6):575-82. DOI: 10.1016/S1470-2045(11)70097-3

4. Kapiteijn E, Kranenbarg EK, Steup WH, Taat CW, Rutten $\mathrm{HJ}$, Wiggers $\mathrm{T}$, et al. Total mesorectal excision (TME) with or without preoperative radiotherapy in the treatment of primary rectal cancer: prospective randomised trial with standard operative and histopathological techniques. Eur J Surg. 1999; 165(5):410-20. DOI:10.1080/110241599750006613.

5. Quirke P. Training and quality assurance for rectal cancer: 20 years of data is enough. Lancet. 2003; 4:695-701. DOI:10.1016/S1470-2045(03)01248-8.

6. PROCARE: multidisciplinary Belgian PROject on CAncer of the Rectum. Multidisciplinary guidelines for the treatment of rectal cancer can be downloaded from www.kankerregister.be (menu: procare) or www.registreducancer.be (menu: procare).

7. AllegraCJ,Yothers G, O'Connell MJ, BeartRW, Wozniak TF, Pitot HC, et al. Neoadjuvant 5-FU or Capecitabine plus radiation with or without oxaliplatin in rectal cancer patients: a phase III randomized clinical trial. J Natl Cancer Inst. 2015;107(11):djv248. DOI:10.1093/ jnci/djv248.

8. Gay HA, Barthold HJ, O'Meara E, Bosch WR, El Naqa I, Al-Lozi R, et al. Pelvic normal tissue contouring guidelines for radiation therapy: a Radiation Therapy Oncology Group consensus panel atlas. Int J Radiat Oncol Biol Phys. 2012;83(3):e353-62.

9. Nagtegaal ID, van Krieken JHJM. The role of pathologists in the quality control of diagnosis and treatment of rectal cancer-an overview. Eur J Cancer 2002;38:964-72

10. Quirke P. The pathologist, the surgeon and colorectal cancer: get it right because it matters. Prog Pathol 1998;4:201-13.

11. Quirke P, Dixon MF, Durdey P, Williams NS. Local recurrence of rectal adenocarcinoma due to inadequate surgical resection: histopathological study of lateral tumour spread and surgical excision. The Lancet. 1986; 328 (8514):996-9. DOI:10.1016/ S0140-6736(86)92612-7

12. Bondeven P. Cancer of the upper rectum. Danish Medical Journal. 2016 Oct; 63(10).

13. Hermanek $P$, Hohenberger $W$, Klimpfinger $M$, et al. The pathological assessment of mesorectal excision: implications for further treatment and quality management. Int J Colorectal Dis. 2003; 18: 33541.

14. Maslekar S, Sharma A, Macdonald A, et al. Mesorectal grades predict recurrences after curative resection for rectal cancer. Dis Colon Rectum. 2007; 50: 16875. DOI:10.1007/s10350-006-0756-2.

15. Nagtegaal ID, van de Velde CJH, van der Worp E, Kapiteijn E, Quirke P, van Krieken JH. Macroscopic evaluation of rectal cancer resection specimen: Clinical significance of the pathologist in quality control. J Clin Oncol. 2002;20(7):1729-34. DOI:10.1200/JCO.2002.07.010. 